\title{
Hypothesis on the Etiology of Skin Complications in Japanese Patients Using Sodium-Glucose Cotransporter 2 Inhibitors
}

\author{
Yoshihiko Suzuki
}

\section{To the Editor}

Skin complications induced by sodium-glucose cotransporter 2 inhibitor (SGLT2i) therapy were frequently reported in Japan from spring to summer in 2014. I also detected such complications in 17 of 142 patients (12\%) treated at my clinic [1]. Drug eruptions usually develop within 4 days to several weeks after initiation of the culprit medication and symptoms tend to vary. However, SGLT2i-induced drug eruptions were particularly varied in 2014 and also attracted attention because of fairly rapid onset after the start of administration.

There are several possible explanations for this outbreak of SGLT2i-induced skin complications in Japan. First, latent allergy to other allergens may have caused drug eruptions that occurred at the initiation of oral SGLT2i administration, with symptoms manifesting due to type I skin allergy. Second, the season might have been the key factor. The incidence of skin complications among patients newly prescribed SGLT2i was lower in autumn and winter. Review of adverse drug reaction information showed that the incidence of drug eruptions was low in these seasons ( $1 \%$ or less), possibly because patients were not affected by pollen or fine particulate matter (PM2.5). The high incidence of skin complications at my clinic in 2014 might have been attributable to prescribing SGLT2 $i$ at the time of the cedar pollen season when PM2.5 levels were also high in Tokyo where the clinic is located. Thus, the characteristic seasonal increase in SGLT2i-induced skin complications suggests that drug eruptions might have developed when induction of dermatitis by cedar pollen and PM2.5 coincided with initiation of SGLT2 $i$ therapy in patients in whom the impact of pollen/PM2.5 had been latent until that time. In fact, several reports support this hypothesis [2-4]. Reports of SGLT2iinduced skin complication increase in spring and summer or from April onward rather than during winter, which contradicts the suggestion that dehydration and dryness are the cause of such eruptions. On the contrary, it is more likely that SGL$\mathrm{T} 2 \mathrm{i}$ will accumulate on the skin surface in a hot sweaty season,

Manuscript accepted for publication January 06, 2017

HDC Atlas Clinic, Atlas Bldg. 1st Floor, 5-3-9 Ichiban-cho, Chiyoda-ku, Tokyo 102-0082, Japan. Email: dr-suzuki@nifty.com

doi: https://doi.org/10.14740/jocmr2897w and induce eruptions after reacting with certain substances [2]. In February 2014, the Beijing City Government issued directives to shut factories judged to be major sources of pollution. Eighteen months later, the National People's Congress approved an amendment to the Law of the People's Republic of China on the Prevention and Control of Atmospheric Pollution with a view to alleviating serious air pollution due to PM2.5. In addition, PM2.5 was included in the indices for monitoring under the Environmental Air Mass Standards (standards for environmental air quality) and the Chinese Ministry of Environmental Protection pledged to conduct comprehensive nationwide monitoring of PM2.5 by 2016. The law enacted in January 2016 allows huge fines (several times larger than before) to be levied by local governments on companies responsible for serious pollution. The number of drug eruptions reported in Japan has been decreasing since these measures were taken. This temporal relationship with events across the East China Sea may be due to chance, and other researchers may have different ideas. Nevertheless, the environmental problem originating in China has been substantiated scientifically, and we have no other plausible theory at hand that adequately explains the skin complications induced by SGLT2i [5]. Accordingly, this hypothesis seems to be worthy of further investigation.

\section{Grant Support}

None.

\section{Disclosure}

I have no conflict of interest and there is no source of funding. I declare that whole manuscript was prepared by myself and I have approved the final version.

\section{References}

1. Suzuki Y. Skin complication of SGLT2 inhibitor treatment in Japanese. Diabetes Res Clin Pract. 2014;106(Supp 1):S265.

2. Takeshita S, Tokunaga T, Tanabe Y, Arinami T, Ichinose T, Noguchi E. Asian sand dust aggregate causes atopic 
dermatitis-like symptoms in Nc/Nga mice. Allergy Asthma Clin Immunol. 2015;11(1):3.

3. Otani S, Onishi K, Mu H, Yokoyama Y, Hosoda T, Okamoto M, Kurozawa Y. The relationship between skin symptoms and allergic reactions to Asian dust. Int J Environ Res Public Health. 2012;9(12):4606-4614.

4. Yokozeki H, Takayama K, Katayama I, Nishioka K. Japanese cedar pollen as an exacerbation factor in atopic dermatitis: results of atopy patch testing and histological examination. Acta Derm Venereol. 2006;86(2):148-151.

5. Mellander A, Billger M, Johnsson E, Traff AK, Yoshida $\mathrm{S}$, Johnsson K. Hypersensitivity Events, Including Potentially Hypersensitivity-Related Skin Events, with Dapagliflozin in Patients with Type 2 Diabetes Mellitus: A Pooled Analysis. Clin Drug Investig. 2016;36(11):925933. 